\title{
Thermally assisted electric field control of magnetism in flexible multiferroic
} heterostructures

MAGNETIC PROPERTIES

AND MATERIALS

FERROELECTRICS AND MULTIFERROICS

Received

7 July 2014

Accepted

17 October 2014

Published

5 November 2014

Correspondence and requests for materials should be addressed to Q.F.Z. (zhanqf@nimte. ac.cn) or R.W.L. (runweili@nimte.ac.cn)

Yiwei Liu, Qingfeng Zhan, Guohong Dai, Xiaoshan Zhang, Baomin Wang, Gang Liu, Zhenghu Zuo, Xin Rong, Huali Yang, Xiaojian Zhu, Yali Xie, Bin Chen \& Run-Wei Li

Key Laboratory of Magnetic Materials and Devices \& Zhejiang Province Key Laboratory of Magnetic Materials and Application Technology, Ningbo Institute of Materials Technology and Engineering (NIMTE), Chinese Academy of Sciences (CAS), Ningbo 315201, People's Republic of China.

Thermal and electrical control of magnetic anisotropy were investigated in flexible $\mathrm{Fe}_{81} \mathrm{Ga}_{19}(\mathrm{FeGa}) /$ Polyvinylidene fluoride (PVDF) multiferroic heterostructures. Due to the large anisotropic thermal deformation of PVDF $\left(\alpha_{1}=-13 \times 10^{-6} \mathrm{~K}^{-1}\right.$ and $\left.\alpha_{2}=-145 \times 10^{-6} \mathrm{~K}^{-1}\right)$, the in-plane uniaxial magnetic anisotropy (UMA) of FeGa can be reoriented $90^{\circ}$ by changing the temperature across $295 \mathrm{~K}$ where the films are magnetically isotropic. Thus, the magnetization of FeGa can be reversed by the thermal cycling between 280 and $320 \mathrm{~K}$ under a constant magnetic field lower than coercivity. Moreover, under the assistance of thermal deformation with slightly heating the samples to the critical temperature, the electric field of \pm $267 \mathrm{kV} \mathrm{cm}^{-1}$ can well align the UMA along the two orthogonal directions. The new route of combining thermal and electrical control of magnetic properties realized in PVDF-based flexible multiferroic materials shows good prospects in application of flexible thermal spintronic devices and flexible microwave magnetic materials.

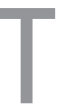

he possibility of controlling magnetism with an electric field, which can be realized by using a kind of multiferroic materials showing coupled ferroelectricity and ferromagnetism, opens a new way for appealing novel magnetization control schemes in future low power consumed spintronic devices ${ }^{1-3}$. However, most of the single-phase multiferroic materials (such as $\mathrm{BiMnO}_{3}, \mathrm{TbMnO}_{3}$ ) exhibit a low Curie temperature and/or a weak intrinsic magnetoelectric (ME) coupling especially above room temperature ${ }^{4,5}$.

$\mathrm{BiFeO}_{3}$ uniquely shows the room temperature $\mathrm{ME}$ coupling, but it is G-type antiferromagnetic or very weak ferromagnetic ${ }^{6,7}$. An attractive alternative way is to use ferromagnetic/ferroelectric (FM/FE) heterostructures, in which electric control of magnetism can be realized through either strain-mediated ME coupling across the interface, exchange bias, or charge-driven ME coupling. Among the three mechanisms, the strain-mediated ME coupling has proven to be more promising ${ }^{8-10}$. In strain-mediated FM/FE heterostructures, a uniaxial strain, which is produced through the converse piezoelectric effect when an electric field applied on FE layer, is transferred to FM layer, due to the inverse magnetostrictive effect, resulting in the change of magnetic properties including magnetic anisotropy, coercive fields, magnetic domains, giant magnetoresistance, etc ${ }^{1-14}$. However, for FM/FE thin-film heterostructures deposited on rigid substrates, the remarkable clamping effect of substrates limit the tunability of magnetic properties by electric field ${ }^{15,16}$. Flexible or freestanding FM/FE heterostructures can reduce or eliminate the substrate clamping effect, thus enhance the ME coupling and the tunability of magnetic properties $^{17-19}$.

Up to now, most of the previously investigated FM/FE multiferroic composites were fabricated by using oxide ferroelectric materials, such as $\mathrm{BaTiO}_{3}, \mathrm{~Pb}(\mathrm{Zr}, \mathrm{Ti}) \mathrm{O}_{3}, \mathrm{PbZn}_{1 / 3} \mathrm{Nb}_{2 / 3} \mathrm{O}_{3}-\mathrm{PbTiO}_{3}$, and $\mathrm{PbMg}_{1 / 3} \mathrm{Nb}_{2 / 3} \mathrm{O}_{3}-$ $\mathrm{PbTiO}_{3}{ }^{20-23}$. However, these oxide ferroelectric materials are extremely fragile and not suitable for preparing flexible or freestanding FM/FE heterostructures ${ }^{24}$. Organic ferroelectric materials, in particular polyvinylidene fluoride (PVDF) and its copolymers, exhibit excellent mechanical flexibility, high piezoelectric voltage constants, and low production cost, which are good candidates for developing flexible multiferroic composites used in magnetic control of polarization ${ }^{25-28}$. For example, flexible SmFe/PVDF heterostructure with a large voltage output of $230 \mu \mathrm{V}$ at $2.3 \mathrm{kOe}$ has been reported to show potential application in weak magnetic-field detection ${ }^{29}$. In contrast, due to the small piezoelectric coefficients $\left(d_{31}=21.4 \mathrm{pC} \mathrm{N}^{-1}, d_{32}=2.3 \mathrm{pC} \mathrm{N}^{-1}\right)$ and the low Young's modulus $(1.5 \mathrm{GPa})$ of $\mathrm{PVDF}^{29,30}$, it seems not possible to achieve an effective electric control of magnetism in 
PVDF based FM/FE heterostructures. On the other hand, PVDF membrane exhibits a large and anisotropic thermal deformation with thermal expansion coefficients $\alpha_{1}=-13 \times 10^{-6} \mathrm{~K}^{-1}$ along the stretch direction during preparation and $\alpha_{2}=-145 \times 10^{-6} \mathrm{~K}^{-1}$ perpendicular to that direction ${ }^{30}$. The characteristics of large thermal deformation have already been used as thermal actuators in microelectro-mechanical-systems ${ }^{31,32}$. In this work, we demonstrate that a significant electric field control of magnetic anisotropy can be realized under assistance of thermal deformation in $\mathrm{Fe}_{81} \mathrm{Ga}_{19} / \mathrm{PVDF}$ heterostructures. The $\mathrm{Fe}_{81} \mathrm{Ga}_{19}$ (FeGa) alloy selected here as the FM layer is a typical magnetostrictive material exhibiting a moderate magnetostriction of $350 \mathrm{ppm}$ under a very low magnetic field and excellent mechanical properties ${ }^{33}$. The multiferroic heterostructure of FeGa/PVDF exhibit a good interfacial coupling, which ensures an efficient transfer of mechanical strain between the two phases. Due to the large anisotropic thermal deformation of PVDF, the uniaxial magnetic anisotropy (UMA) can be reoriented in the plane of FeGa films by varying the temperature. Under a constant magnetic field, the magnetization of FeGa can be reversed by thermal cycling across the critical temperature of $295 \mathrm{~K}$ where FeGa films are magnetically isotropic. In addition, the combined electric field and thermal deformation can lead to the reorientation of UMA in FeGa/ PVDF films through a strain-mediated converse ME coupling.

\section{Results}

FeGa films with $60 \mathrm{~nm}$ in thickness were deposited by magnetronsputtering at an ambient temperature of $291 \mathrm{~K}$ on $30-\mu \mathrm{m}$-thick PVDF membrane coated with 50-nm-thick Al layers on both sides. A $5 \mathrm{~nm}$ Au layer was deposited to protect against oxidation. The sample structure is schematically shown in Figure 1a. We define in the plane of PVDF membrane that $d_{31}$ and $\alpha_{1}$ are along the $x$ direction, and $d_{32}$ and $\alpha_{2}$ are along the $y$ direction. The XRay diffraction measurement confirms that the PVDF membrane is $\beta$-phase which possesses the ferroelectricity (Figure S1) ${ }^{34}$. The magnetic hysteresis loops of FeGa/PVDF films were measured at different temperatures by a superconducting quantum interference device-vibrating sample magnetometer (SQUID-VSM) and at different applied electric fields by a magneto-optical Kerr effect (MOKE) setup. During the magnetic measurements, an in-plane magnetic field $H$ was applied at an angle of $\theta$ with respect to the $x$ direction (Figure 1a).

The hysteresis loop of the as-prepared FeGa/PVDF film measured along the $x$ direction at $291 \mathrm{~K}$ exhibits a relative slanted shape with a $M_{\mathrm{r}} / M_{\mathrm{s}}$ ratio of 0.65 (Figure $1 \mathrm{~b}$ ), while the corresponding hysteresis loop measured along the $y$ direction is square with a $M_{\mathrm{r}} / M_{\mathrm{s}}$ ratio of 0.91 (Figure 1c), where $M_{\mathrm{r}}$ and $M_{\mathrm{s}}$ are the remanent and saturation magnetization, respectively. These magnetic features indicate that the FeGa/PVDF film shows a UMA along the $y$ direction. When the temperature increases to $295 \mathrm{~K}$, the $M_{\mathrm{r}} / M_{\mathrm{s}}$ ratios for $H$ along the $x$ and $y$ directions are nearly identical, which indicates that the FeGa/PVDF film is magnetically isotropic at this critical temperature. At $300 \mathrm{~K}$, the $M_{\mathrm{r}} / M_{\mathrm{s}}$ ratio of 0.93 along the $x$ direction becomes remarkably greater than that of 0.43 along the $y$ direction, which indicates that the easy magnetization direction of FeGa/PVDF film is reoriented from the $y$ direction at $291 \mathrm{~K}$ to the $x$ direction at $300 \mathrm{~K}$. The additional angular dependent magnetic measurements confirm that the FeGa/PVDF film at the critical temperature of $295 \mathrm{~K}$ is magnetically isotropic with $M_{\mathrm{r}} / M_{\mathrm{s}}$ of 0.82 at arbitrary orientation of magnetic field (Figure 1d). The symmetries of the angular dependence of $M_{\mathrm{r}} / M_{\mathrm{s}}$ ratios obtained at 291 and $300 \mathrm{~K}$ confirm that the UMA in FeGa film below and above the critical temperature are oriented along the $y$ and $x$ directions, respectively (Figure $1 \mathrm{~d}$ ). We ascribe the origin of UMA and the change with temperature to the anisotropic thermal deformation of PVDF. Although we did not set a substrate temperature during the deposition of FeGa layer, the heating effect of sputtered atoms gives rise to a slight thermal contraction of PVDF. After cooling down to the ambient temperature of $291 \mathrm{~K}$, the difference in the thermal expansion of PVDF between the $x$ and $y$ directions results in an uniaxial tensile strain $\varepsilon_{\mathrm{T}}=k_{c}\left(\alpha_{1}-\alpha_{2}\right) \Delta T$ along the $y$ direction, which can be transferred to FeGa layer across the interface. The interfacial coupling factor $k_{c}\left(0 \leq k_{c} \leq 1\right)$ results from the clamping effect of metallic layers on PVDF, which depends on the Young's moduli, the thicknesses of each layer (including ferromagnetic FeGa layer, ferroelectric PVDF membrane, and two Al electrodes), and the interfacial bondings between the neighboring layers ${ }^{35}$. The Young's moduli of metallic layers $\left(E_{f}=60 \mathrm{GPa}\right.$ for FeGa and $E_{a}$ $=66 \mathrm{GPa}$ for $\mathrm{Al})$ are about 40 times larger than that of $\mathrm{PVDF}\left(E_{p}=\right.$ $1.5 \mathrm{GPa})^{29,36}$. The metallic layers with a comparable thickness would
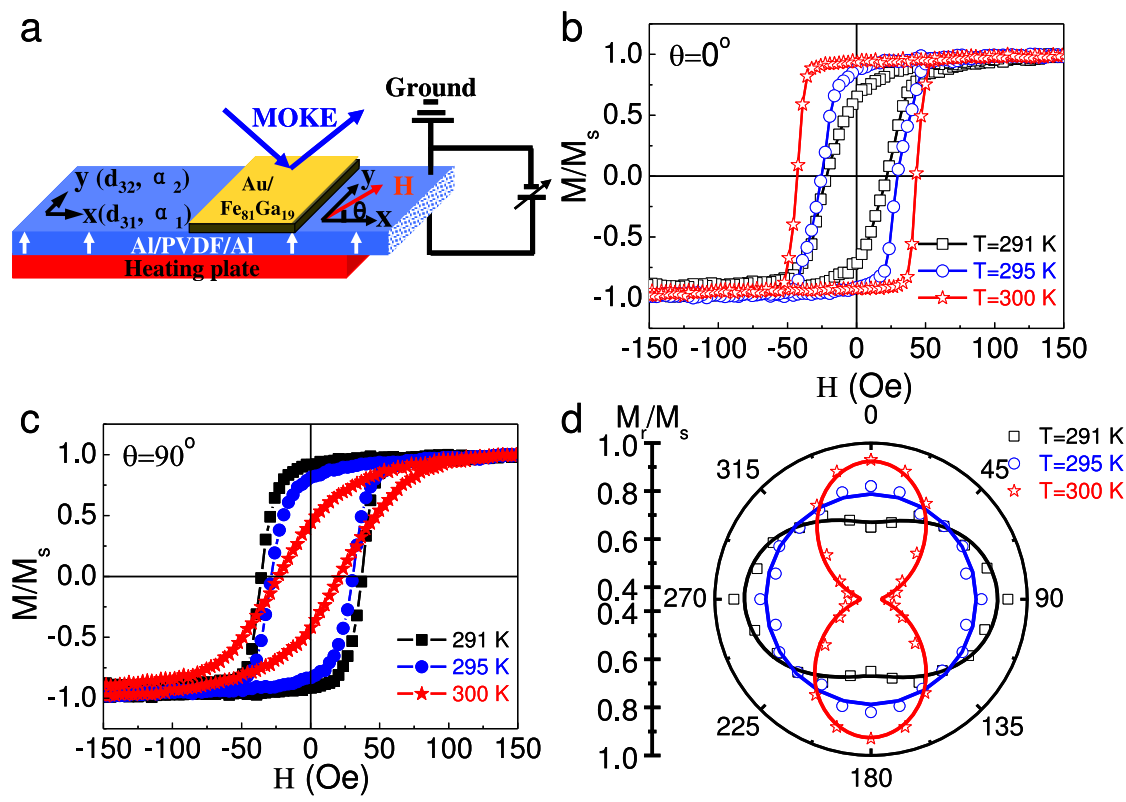

Figure $1 \mid$ (a) Schematic view of the sample structure of FeGa/PVDF heterostructures and the configuration of magnetic measurement. Magnetic hysteresis loops of FeGa/PVDF film measured with an in-plane magnetic field applied along (b) the $x \operatorname{direction}\left(\theta=0^{\circ}\right)$ and $(\mathrm{c})$ the $y$ direction $\left(\theta=90^{\circ}\right)$ at various temperatures. (d) Angular dependence of normalized remanent magnetization for FeGa/PVDF film at different temperatures. 

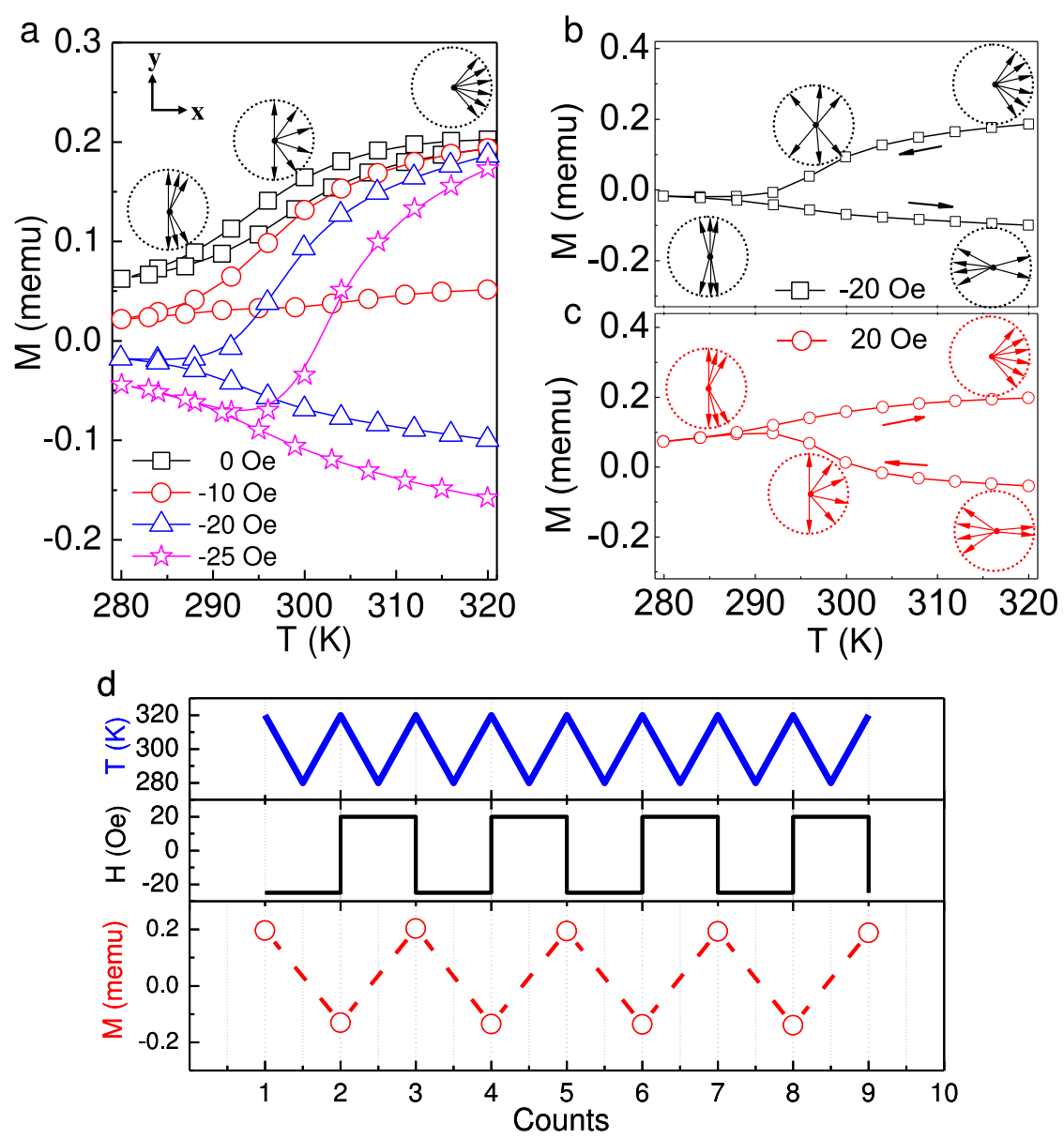

Figure 2 $\mid$ Temperature dependence of magnetization for FeGa/PVDF film in cooling and warming processes between 280 and $320 \mathrm{~K}$ under (a) different magnetic fields ranging from 0 to $-25 \mathrm{Oe}$, (b) $-20 \mathrm{Oe}$, and (c) 20 Oe. The orientations of FeGa domains during thermal cycling under 0 , -20 , and 20 Oe are schematically shown in the insets of a, b, and c, respectively. (d) Magnetization of FeGa/PVDF film reversed by thermal cycling under an alternatively positive and negative magnetic field. Top: thermal cycles between 280 and $320 \mathrm{~K}$. Middle: an alternatively positive and negative magnetic field used for the measurements. Bottom: measurements of magnetization under the sequence of thermal cycles and magnetic field.

significantly clamp the deformation of PVDF with changing temperature. However, in our samples, the thickness of PVDF $\left(t_{p}=30 \mu \mathrm{m}\right)$ is two orders in magnitude larger than the total thickness of both $\mathrm{FeGa}\left(t_{f}=60 \mathrm{~nm}\right)$ and $\mathrm{Al}\left(t_{a}=100 \mathrm{~nm}\right)^{37}$, which leads to a theoretical value of $k_{c}$ about $81.5 \%$ by using the relation of $k_{c}=\frac{E_{p} t_{p}}{E_{p} t_{p}+E_{a} t_{a}+E_{f} t_{f}}$ (Supporting information, Figure S2) ${ }^{38}$. Good and tight chemical bondings between the neighboring layers can ensure that the experimental value of $k_{c}$ is close to the theoretical value. Consequently, due to the inverse magnetostriction effect of FeGa film, an UMA $K_{\mathrm{T}}=3 / 2 \lambda_{\mathrm{s}} \sigma_{\mathrm{T}}$ along the $y$ direction can be induced when the as-prepared FeGa/PVDF film is cooled down, where $\lambda_{\mathrm{s}}$ is the magnetostriction constant of FeGa film. The stress applied on FeGa film is $\sigma_{\mathrm{T}}=\varepsilon_{\mathrm{T}} E_{f} /\left(1-v^{2}\right)^{36}$, where $v$ is the Poisson ratio of FeGa film. With increasing temperature from below to above the critical temperature, the thermal contraction along the $y$ direction would reverse the strain imposed on FeGa film from tensile to compressive, leading to the reorientation of UMA from the $y$ to $x$ directions. Similarly, decreasing the temperature across the critical temperature may also result in the inverse reorientation of UMA from the $x$ to $y$ directions. The further increasing or decreasing temperature away from the critical point would enhance the strength of UMA.

The thermally induced reorientation of UMA in FeGa/PVDF films can be employed to reverse the magnetization under a constant magnetic field. Before thermal cycling, the FeGa film is fully magnetized along the $x$ direction by a saturation magnetic field of $200 \mathrm{Oe}$ at $320 \mathrm{~K}$. Figure 2a shows the temperature dependence of magnetization by cooling and warming the FeGa/PVDF film between 320 and $280 \mathrm{~K}$ with applying different magnetic fields from 0 to -25 Oe along the $x$ direction. The magnetic fields are selected to be smaller than the measured coercive fields which are $27 \mathrm{Oe}$ at $280 \mathrm{~K}$ and $44 \mathrm{Oe}$ at $320 \mathrm{~K}$ (Supporting information, Figure S3). With cooling the sample without magnetic field from 320 to $280 \mathrm{~K}$, the magnetization of FeGa film decays about 68 percent from 0.19 to 0.06 memu. In the following warming process from 280 to $320 \mathrm{~K}$, the magnetization returns to the original value. It should be noted that the spontaneous magnetization of $\mathrm{FeGa}$ is oppositely increased about 2.8 percent for the temperature varied from 320 to $280 \mathrm{~K}$ (Supporting information, Figure S4), which is far less than the strain effect caused by the thermal deformation of PVDF. A negative magnetic field applied during thermal cycling results in a significant difference of magnetization between before and after thermal cycling. The increase in the strength of magnetic field may enlarge this difference and leads to the magnetization after thermal cycling changing from 0.05 to -0.16 memu for the magnetic field increasing from -10 to -25 Oe. As shown in Figure $2 b$, the thermal cycling with a negative magnetic field of -20 Oe can switch the magnetization of FeGa films from 0.19 to -0.16 memu. The following thermal cycle with a positive magnetic field of 20 Oe may reverse the magnetization back to 0.19 memu (Figure 2c). In Figure 2d, we demonstrate that the thermal cycling associated with an alternatively positive and 

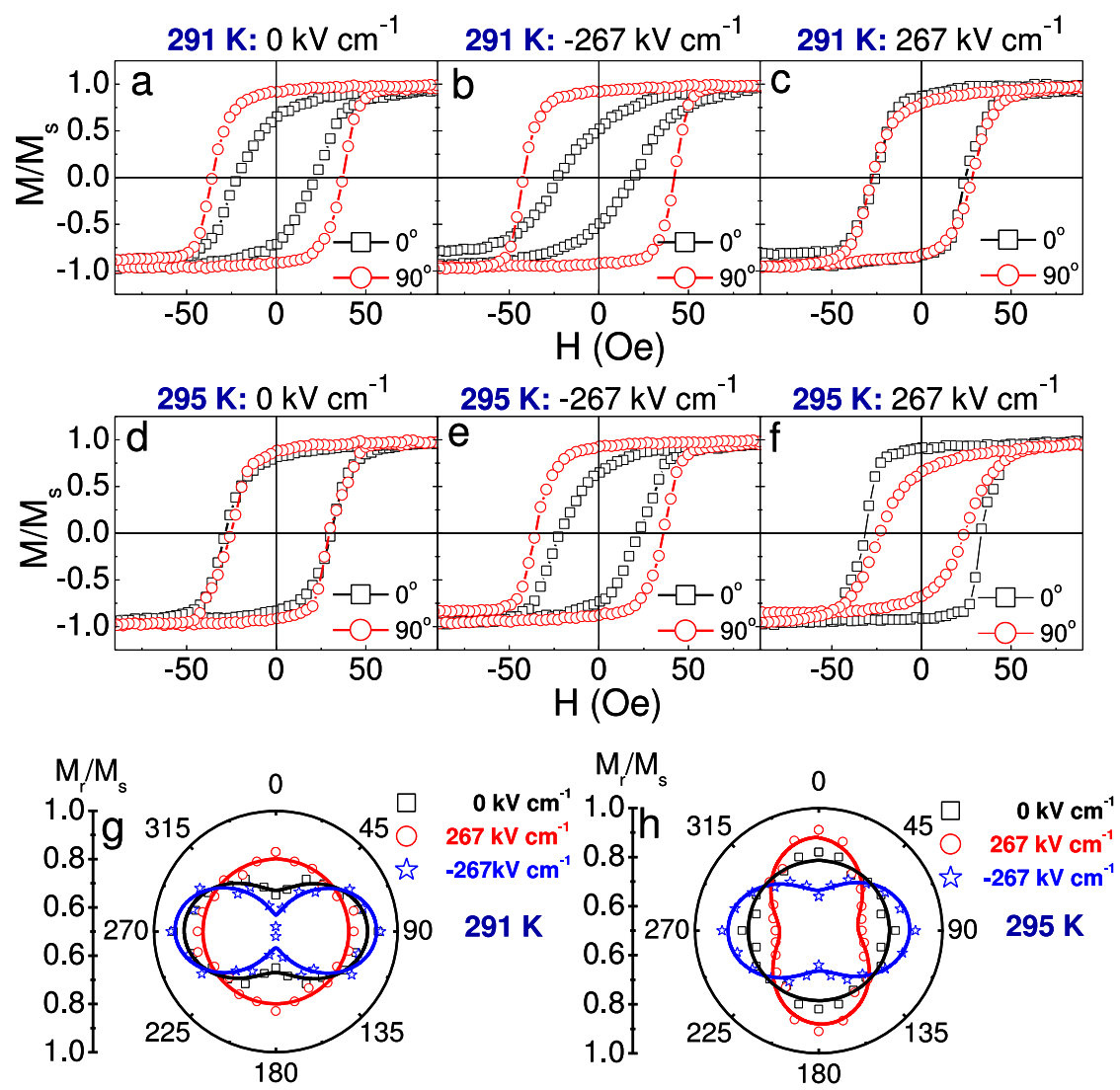

Figure 3 Thermally assisted electric field control of the UMA in FeGa/PVDF film. In-plane magnetic hysteresis loops of FeGa/PVDF film measured at $291 \mathrm{~K}$ with (a) $\mathrm{E}=0 \mathrm{kV} \mathrm{cm}^{-1}$, (b) $E=-267 \mathrm{kV} \mathrm{cm}^{-1}$, and (c) $E=267 \mathrm{kV} \mathrm{cm}^{-1}$ applied through the thickness of PVDF. In-plane magnetic hysteresis loops measured at $295 \mathrm{~K}$ with (d) $E=0 \mathrm{kV} \mathrm{cm}^{-1}$, (e) $E=-267 \mathrm{kV} \mathrm{cm}^{-1}$, and (f) $E=267 \mathrm{kV} \mathrm{cm}^{-1}$. The corresponding angular dependence of normalized remnant magnetization of FeGa/PVDF film measured at (g) $291 \mathrm{~K}$ and (h) $295 \mathrm{~K}$ with different electric fields applied on PVDF.

negative magnetic field can switch the magnetization of FeGa/PVDF film between the positive and negative directions.

The magnetization reversed by the thermal cycling under the assistance of magnetic field can be understood with considering the change of domain orientations in $\mathrm{FeGa} / \mathrm{PVDF}$ with temperature, as schematically shown in the insets of Figure 2 a to $2 \mathrm{c}$. At $320 \mathrm{~K}$, the orientations of magnetic domains in FeGa layer are squeezed into a narrow distribution around the positive direction of UMA along the $x$ direction by a saturation magnetic field. With zero-field cooling the sample, the strength of UMA is reduced due to the tensile strain along the $y$ direction produced by the thermal expansion of PVDF. Consequently, the distribution of domain orientations becomes much broader. At the critical point of $295 \mathrm{~K}$ where FeGa/PVDF film is magnetically isotropic, the domain orientations of FeGa distribute arbitrarily in the film plane with components along the positive $x$ direction. The further decrease of temperature results in the enhancement of the reoriented UMA along the $y$ direction and squeezes the FeGa domain orientations along both the positive and negative $y$ directions. Therefore, the magnetization of FeGa film measured along the $x$ direction decreases in the zero-field cooling process. When warming the sample back to $320 \mathrm{~K}$, the reorientation of UMA can drive the FeGa domain orientations back to the original state of narrow distribution along the $x$ direction. In case of applying a negative magnetic field during thermal cycling, the magnetic field rotates $\mathrm{FeGa}$ domain orientations towards the negative $x$ direction (Figure 2b). The domain orientations with the negative $x$ component, which amount depends on the strength of applied field, would be squeezed into a narrow distribution along the negative $x$ direction after a thermal cycle of cooling and warming processes across the critical temperature of magnetic isotropy. It is noted that since the applied magnetic field is lower than the coercivity, not all of the magnetization can be switched from positive to negative after thermal cycling under a negative magnetic field. In contrast, a following thermal cycle under an identical positive field can reverse all the magnetization with negative component to positive (Figure 2c).

The electric field control of magnetic properties in FeGa/PVDF film was measured by a MOKE magnetometer with an electric field applied through the thickness of PVDF. At the ambient temperature of $291 \mathrm{~K}$, the Kerr hysteresis loops measured at different orientations of magnetic field indicate that the as-prepared $\mathrm{FeGa} / \mathrm{PVDF}$ sample possesses a UMA along the $y$ direction (Figure $3 \mathrm{a}$ ). When applying an electric field on PVDF, the remanent magnetization of FeGa measured along the $x$ and $y$ directions are increased and decreased linearly with increasing the electric field from -267 to $267 \mathrm{kV} \mathrm{cm}^{-1}$ due to the strain-mediated converse ME coupling, respectively (Supporting information, Figure S5). Except a slight change in $M_{\mathrm{r}}$ / $M_{\mathrm{s}}$, the hysteresis loops measured at $E=-267 \mathrm{kV} \mathrm{cm}^{-1}$ are similar to that obtained without external electric field, which suggests that the UMA of FeGa layer remains aligned along the $y$ direction under a negative electric field, but the strength is slightly enhanced (Figure $3 \mathrm{~b}$ ). However, when $E=267 \mathrm{kV} \mathrm{cm}^{-1}$, the hysteresis loops measured along both the $x$ and $y$ directions are almost identical, which indicates a magnetic isotropy in FeGa/PVDF film (Figure 3c). The angular dependent magnetic measurements confirm the changes of UMA in FeGa/PVDF at various electric fields (Figure 3g). It is clearly seen that at $T=291 \mathrm{~K}$ the strain-mediated converse ME coupling cannot lead to a reorientation of UMA in FeGa layer by applying an electric field on PVDF membrane due to the rather small piezoelectric coefficients of PVDF and the UMA in FeGa layer. 

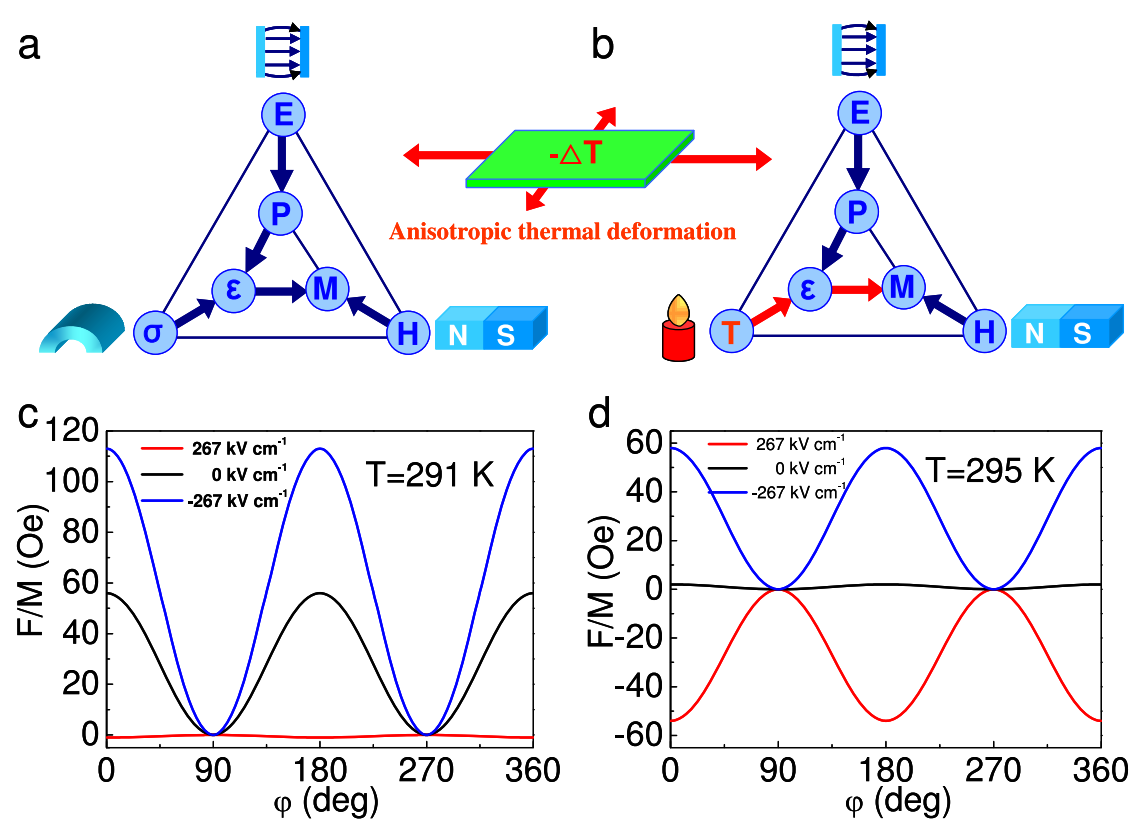

Figure $4 \mid$ (a) Schematic show of conventional electric field control of magnetism in FM/FE multiferroic composites. (b) A new route of thermally assisted electric field control of magnetism realized in FeGa/PVDF heterostructures based on the anisotropic thermal deformation of PVDF. Energy landscapes of FeGa/PVDF film at (c) $291 \mathrm{~K}$ and (d) $295 \mathrm{~K}$ under different electric fields applied on PVDF.

In the present PVDF based heterostructures, we demonstrate that the thermal deformation can be used to eliminate the UMA in FeGa layer and assist electric field to realize the reorientation of UMA in $\mathrm{FeGa} / \mathrm{PVDF}$ film. When we increase the temperature to $295 \mathrm{~K}$, the hysteresis loops measured with a magnetic field applied along the $x$ and $y$ directions at $E=0$ show that the FeGa/PVDF film is almost magnetically isotropic (Figure $3 \mathrm{~d}$ ), which indicates that the UMA is remarkably reduced by slightly increasing the temperature through the effect of anisotropic thermal contraction. When applying an electric field of $-267 \mathrm{kV} \mathrm{cm}{ }^{-1}$ on PVDF, the $M_{\mathrm{r}} / M_{\mathrm{s}}$ ratio measured along the $y$ direction becomes larger than that obtained along the $x$ direction (Figure 3e). On the contrary, for $E=267 \mathrm{kV} / \mathrm{cm}$, the $M_{\mathrm{r}} /$ $M_{\mathrm{s}}$ ratio along the $y$ direction is smaller than that along the $x$ direction (Figure 3f). Obviously, after warming to $295 \mathrm{~K}$, depending on the electric field applied on PVDF, the UMA of FeGa layer can be adjusted along the $x$ or $y$ directions. The in-plane reorientation of UMA driven by electric field can be clearly seen in the angular dependence of normalized remanent magnetization measured at $T=295 \mathrm{~K}$ with different electric fields applied on PVDF (Figure $3 \mathrm{~h}$ ).

Figure 4a shows the conventional strain-mediated mechanism for electric control of magnetism in FM/FE multiferroic heterostructures. Due to the converse piezoelectric effect, an electric field applied on FE phase produces a strain which can be transferred to FM phase. Then, the magnetic properties of FM can be modified by this strain through the inverse magnetostrictive effect. For FM/FE heterostructures using PVDF as the ferroelectric layer, thanks to the characteristics of large anisotropic thermal deformation of PVDF, a variation of temperature may additionally generate a mechanical strain, assisting electric field to change the magnetic properties of FM phase, which provides us a new route for multi-field control of magnetism in FM/FE heterostructures (Figure $4 \mathrm{~b}$ ).

\section{Discussion}

In order to quantitatively understand the thermally assisted electric field control of magnetic anisotropy in FeGa/PVDF film, the total free energy density $F$ of $\mathrm{FeGa}$ film can be written as: $F=\left(K_{\mathrm{E}}+K_{\mathrm{T}}\right) \cos ^{2} \varphi-M H \cos (\theta-\varphi)$, where $K_{\mathrm{E}}$ and $K_{\mathrm{T}}$ are the UMAs induced by the electric field through the converse piezoelectric effect and by the variation of temperature through the anisotropic thermal deformation effect, respectively, and $\varphi$ is the angle between the magnetization and the $x$ direction. Due to the dimensions of FeGa films, the in-plane demagnetization energy is too weak to be taken into account $^{39}$, which only provides a negligible constant term into the free energy. $K_{\mathrm{E}}$ depends on the piezoelectric coefficients of PVDF and the applied electric field. Thus, $K_{\mathrm{E}}=3 \varepsilon_{\mathrm{E}} \lambda_{s} E_{f} / 2\left(1-v^{2}\right)$, where the electric-field-induced strain $\varepsilon_{\mathrm{E}}$ is evaluated to be $k_{c}\left(d_{31}-d_{32}\right) E . \lambda_{\mathrm{s}}$ and $E_{f}$ are estimated to be about $100 \mathrm{ppm}$ and $60 \mathrm{GPa}$ for polycrystalline FeGa film, respectively ${ }^{36} . v$ is chosen to be a typical value of 0.3 for metals ${ }^{40}$. We assume $K_{\mathrm{T}}=0$, i.e., magnetic isotropy for PVDF at $295 \mathrm{~K}$. According to these parameters, the energy landscapes of FeGa/PVDF film under different applied electric fields at 291 and $295 \mathrm{~K}$ under zero magnetic field can be numerically calculated, as respectively shown in Figure $4 \mathrm{c}$ and $4 \mathrm{~d}$. At $291 \mathrm{~K}$, the FeGa film shows the UMA along the $y$ direction, which is originated from the strain caused by the anisotropic thermal deformation of PVDF. A uniaxial strain produced by an electric field of $267 \mathrm{kV} \mathrm{cm}^{-1}$ applied on PVDF via the converse piezoelectric effect can greatly reduce the strength of UMA, but not reverse the orientation of UMA in $\mathrm{FeGa}$ / PVDF. As a contrast, a negative electric field of $-267 \mathrm{kV} \mathrm{cm}^{-1}$ may enhance the UMA along the $y$ direction. The slight increase of temperature to $295 \mathrm{~K}$ generates a compressive strain along the $y$ direction due to the anisotropic thermal contraction, and reduces the energy barrier of UMA. Consequently, in this case, a positive electric field applied on PVDF is able to reorient the UMA of FeGa along the $x$ direction, while a negative electric field enhances the UMA along the $y$ direction, which are consistent with the experimental observations shown in Figure 3.

In conclusion, we have successfully fabricated flexible multiferroic $\mathrm{FeGa} / \mathrm{PVDF}$ heterostructures. Due to the large anisotropic thermal deformation of the PVDF membrane, the in-plane UMA of FeGa layer can be reoriented $90^{\circ}$ by changing temperature across $295 \mathrm{~K}$ where FeGa films are magnetically isotropic. Utilizing this special characteristic, the magnetization of FeGa can be reversed by thermal cycling between 280 and $320 \mathrm{~K}$ under a constant magnetic field lower than the coercivity. Because of the rather small piezoelectric coefficients, a sufficient electric field applied on PVDF cannot lead to a reorientation of UMA in FeGa at the ambient temperature of $291 \mathrm{~K}$. Under the assistance of thermal deformation by slightly heating the 
sample to $295 \mathrm{~K}$, the electric field of $\pm 267 \mathrm{kV} \mathrm{cm}^{-1}$ is able to align the UMA along the two orthogonal directions through a strainmediated converse ME coupling. Our experimental results suggest that the thermal control of magnetic properties based on the anisotropic thermal deformation of PVDF have promising applications in thermally tunable or thermally assisted electric field tunable flexible spintronic devices and flexible microwave magnetic materials.

\section{Methods}

Specimen Fabrication. The commercial PVDF membranes with $30 \mu \mathrm{m}$ in thickness from were sputtered with 50-nm-thick Al layers on both sides. The $60 \mathrm{~nm}$ FeGa layers were deposited onto PVDF at an ambient temperature of $291 \mathrm{~K}$ by using a magnetron sputtering system with a base pressure better than $5 \times 10^{-5} \mathrm{~Pa}$. During sputtering, the argon flow was kept at $50 \mathrm{sccm}$ and the pressure was set at $1.0 \mathrm{~Pa}$. The growth rate of FeGa film was controlled at $10.0 \mathrm{~nm} \mathrm{~min}{ }^{-1}$. A deposition rate of $2.0 \mathrm{~nm} \mathrm{~min}{ }^{-1}$ was used for growing a $5 \mathrm{~nm}$ Au protection layer.

Characterization. The crystalline structure of PVDF was checked by an $\mathrm{x}$-ray diffraction meter (D8 Advance, Bruker) with $\mathrm{Cu} \mathrm{K} \alpha$ radiation. The thicknesses of FeGa and Au layers were calibrated by X-ray reflectivity. A standardized ferroelectric test system (Precision Premier II, Radiant Technologies) was used to measure the electric hysteresis loops of PVDF. A superconducting quantum interference devicevibrating sample magnetometer (Quantum Design) was employed to measure the magnetic hysteresis loops of $\mathrm{FeGa} / \mathrm{PVDF}$ in the temperature range from 280 to $320 \mathrm{~K}$. The angular dependence of magnetic hysteresis loops of FeGa layer was measured at different polarization states of PVDF by magneto-optical Kerr effect (MOKE) from 291 to $300 \mathrm{~K}$. A hot plate mounted on the back of samples was used to heat the samples during MOKE measurements. The Al layers on both sides of PVDF were connected to a voltage source (Keithley 237 High-Voltage Source-Measure Unit) with thin Pt wires. During the MOKE measurements, the voltage source provides an electric field to polarize the ferroelectric PVDF membrane thought the thickness.

1. Eerenstein, W. et al. Multiferroic and magnetoelectric materials. Nature (London) 442, 759-765 (2006).

2. Fiebig, M. Revival of the magnetoelectric effect. J. Phys. D: Appl. Phys. 38, R123-R152 (2005)

3. Spaldin, N. A. et al. The renaissance of magnetoelectric multiferroics. Science 309, 391-392 (2005).

4. Cheong, S. W. et al. Multiferroics: A magnetic twist for ferroelectricity. Nat. Mater. 6, 13-20 (2007)

5. Khomskii, D. Classifying multiferroics: Mechanisms and effects. Physics 2, 20 (2009).

6. Lebeugle, D. et al. Electric-field-induced spin flop in $\mathrm{BiFeO}_{3}$ single crystals at room temperature. Phys. Rev. Lett. 100, 227602 (2008).

7. Lee, S. et al. Electric field control of the magnetic state in $\mathrm{BiFeO}_{3}$ single crystals. Appl. Phys. Lett. 92, 192906 (2008).

8. Eerenstein, W. et al. Giant sharp and persistent converse magnetoelectric effects in multiferroic epitaxial heterostructures. Nat. Mater. 6, 348-351 (2007).

9. Ma, J. et al. Recent progress in multiferroic magnetoelectric composites: From bulk to thin films. Adv. Mater. 23, 1062-1087 (2011).

10. Lahtinen, T. H. E. et al. Pattern transfer and electric-field-induced magnetic domain formation in multiferroic heterostructures. Adv. Mater. 23, 3187-3191 (2011).

11. Weiler, M. et al. Voltage controlled inversion of magnetic anisotropy in a ferromagnetic thin film at room temperature. New J. Phys. 11, 013021 (2009).

12. Li, Z. et al. A magnetoelectric memory cell with coercivity state as writing data bit. Appl. Phys. Lett. 96, 162505 (2010).

13. Zavaliche, F. et al. Electric field-induced magnetization switching in epitaxial columnar nanostructures. Nano Lett. 5, 1793-1796 (2005).

14. Liu, M. et al. Electric field modulation of magnetoresistance in multiferroic heterostructures for ultralow power electronics. Appl. Phys. Lett. 98, 222509 (2011)

15. Zheng, $\mathrm{H}$. et al. Multiferroic $\mathrm{BaTiO}_{3}-\mathrm{CoFe}_{2} \mathrm{O}_{4}$ nanostructures. Science 303, 661-663 (2004)

16. Dokupil, S. et al. Positive/negative magnetostrictive GMR trilayer systems as strain gauges. J. Magn. Magn. Mater. 795, 290-291 (2005).

17. Brintlinger, T. et al. In situ observation of reversible nanomagnetic switching induced by electric fields. Nano Lett. 10, 1219-1223 (2010).

18. Marauska, S. et al. MEMS magnetic field sensor based on magnetoelectric composites. J. Micromech. Microeng. 22, 065024 (2012).

19. Nan, T. et al. Magnetoelectric NEMS Resonator for Ultra-Sensitive DC Magnetic Field Detection. Sci. Rep. 3, 1985 (2013).

20. Thiele, C. et al. Influence of strain on the magnetization and magnetoelectric effect in $\mathrm{La}_{0.7} \mathrm{~A}_{0.3} \mathrm{MnO}_{3} / \mathrm{PMN}-\mathrm{PT}(001)(\mathrm{A}=\mathrm{Sr}, \mathrm{Ca})$. Phys. Rev. B 75, 054408 (2007).
21. Yang, J. J. et al. Electric field manipulation of magnetization at room temperature in multiferroic $\mathrm{CoFe}_{2} \mathrm{O}_{4} / \mathrm{Pb}\left(\mathrm{Mg}_{1 / 3} \mathrm{Nb}_{2 / 3}\right)_{0.7} \mathrm{Ti}_{0.3} \mathrm{O}_{3}$ heterostructures. Appl. Phys. Lett. 94, 212504 (2009)

22. Wang, J. et al. Large Electric-Field Modulation of Magnetic Properties in Fe Films on BiScO3-PbTiO3 Ceramics. J. Nanomater. 2010, 142750 (2010).

23. Sahoo, S. et al. Ferroelectric control of magnetism in BaTiO3/Fe heterostructures via interface strain coupling. Phys. Rev. B 76, 092108 (2007).

24. Dakua, I. \& Afzulpurkar, N. Piezoelectric Energy Generation and Harvesting at the Nano-Scale: Materials and Devices. Nanomater. Nanotechnol. 3, 1-16 (2013).

25. Kharat, D. K. et al. Polymeric piezoelectric transducers for hydrophone applications. Defence. Sci. J. 57, 7-22 (2007).

26. Jayakumar, O. D. et al. Inorganic-organic multiferroic hybrid films of $\mathrm{Fe}_{3} \mathrm{O}_{4}$ and PVDF with significant magneto-dielectric coupling. J. Mater. Chem. C 1, 3710-3715 (2013).

27. Martins, P. et al. Large linear anhysteretic magnetoelectric voltage coefficients in $\mathrm{CoFe}_{2} \mathrm{O}_{4}$ /polyvinylidene fluoride 0-3 nanocomposites. J. Nanopart. Res. 15, 1825 (2013).

28. Martins, P. et al. Interface characterization and thermal degradation of ferrite/ poly(vinylidene fluoride) multiferroic nanocomposites. J. Mater. Sci. 48, 2681-2689 (2013).

29. Zhao, S. F. et al. Flexible Sm-Fe/polyvinylidene fluoride heterostructural film with large magnetoelectric voltage output. Appl. Phys. Lett. 97, 212902 (2010).

30. Chang, H. H. S. et al. Pyroelectric effect enhancement in laminate composites under short circuit condition. J. Appl. Phys. 106, 114110 (2009).

31. Park, J. S. et al. Bent-beam electrothermal actuators-Part II: Linear and rotary microengines. J. Microelectromech. S. 10, 255-262 (2001).

32. Maloney, J. M. et al. Large force electro thermal linear micromotors. J. Micromech. Microeng. 14, 226-234 (2004).

33. Clark, A. E. et al. Magnetostrictive properties of body-centered cubic Fe-Ga and $\mathrm{Fe}-\mathrm{Ga}-\mathrm{Al}$ alloys. IEEE Trans. Magn. 36, 3238-3240 (2000).

34. Lovinger, A. J. et al. Crystallographic changes characterizing the Curie transition in three ferroelectric copolymers of vinylidene fluoride and trifluoroethylene: 1 . As-crystallized samples. Polymer 24, 1225-1232 (1983).

35. Nan, C. W. et al. Multiferroic magnetoelectric composites: Historical perspective, status, and future directions. J. Appl. Phys. 103, 031101 (2008).

36. Dai, G. H. et al. Mechanically tunable magnetic properties of $\mathrm{Fe}_{81} \mathrm{Ga}_{19}$ films grown on flexible substrates. Appl. Phys. Lett. 100, 122407 (2012).

37. Noble, B. et al. The elastic modulus of Al-Li alloys. J. Mater. Sci. 17, 461-468 (1982).

38. Yang, F. Q. \& Li, J. C. M. Diffusion-induced beam bending in hydrogen sensors. J. Appl. Phys. 93, 9304 (2003).

39. Aharoni, A. Demagnetizing factors for rectangular ferromagnetic prisms. J. Appl. Phys. 83, 3432 (1998)

40. Lei, N. et al. Magnetization reversal assisted by the inverse piezoelectric effect in Co-Fe-B/ferroelectric multilayers. Phys. Rev. B 84, 012404 (2011).

\section{Acknowledgments}

This work was supported by the National Natural Science Foundation of China (11304326, $11174302,11374312,11274321)$ the State Key Project of Fundamental Research of China (2012CB933004), the Ningbo Science and Technology Innovation Team (2011B82004), and Ningbo Natural Science Foundations (2013A610083)

\section{Author contributions}

Y.L., Q.Z. and R.L. conceived the experiments. Y.L., G.D. and X.Z. carried out experiments Y.L. and Q.Z. did modeling. Y.L., Q.Z. and R.L. wrote the manuscript. B.W., G.L., Z.Z., X.R. H.Y., X.Z., Y.X. and B.C. discussed the data and the results, and commented the manuscript

\section{Additional information}

Supplementary information accompanies this paper at http://www.nature.com/ scientificreports

Competing financial interests: The authors declare no competing financial interests.

How to cite this article: Liu, Y. et al. Thermally assisted electric field control of magnetism in flexible multiferroic heterostructures. Sci. Rep. 4, 6925; DOI:10.1038/srep06925 (2014).

This work is licensed under a Creative Commons Attribution-NonCommercialShareAlike 4.0 International License. The images or other third party material in this article are included in the article's Creative Commons license, unless indicated otherwise in the credit line; if the material is not included under the Creative Commons license, users will need to obtain permission from the license holder in order to reproduce the material. To view a copy of this license, visit http:// creativecommons.org/licenses/by-nc-sa/4.0/ 